A. van der Gaast $\cdot$ J. Verweij $\cdot$ A. S. Th. Planting

G. Stoter - S. C. Henzen-Logmans

\title{
The value of immunohistochemistry in patients with poorly differentiated adenocarcinomas and undifferentiated carcinomas of unknown primary
}

Received: 18 February 1995/Accepted: 26 August 1995

\begin{abstract}
A subgroup of patients with metastatic carcinomas of unknown origin may benefit from combination chemotherapy. The relevance of immunohistochemistry in detecting such patients was investigated. Immunohistochemical studies with a panel of antibodies were performed on the tissue specimens of 41 patients having a light-microscopic diagnosis of poorly differentiated adenocarcinoma or undifferentiated carcinoma of unknown origin, who had been treated with cisplatin-containing chemotherapy. The study aimed to answer the following questions: (a) Can the tissue type of the tumor be verified? (b) Can a primary organ site be identified? (c) Can a prognostic immunohistochemical profile be recognized? The original diagnosis had to be changed in 2 of the 41 patients, who turned out to have a malignant lymphoma and neuroblastoma, respectively. The primary site was diagnosed in a patient with prostate cancer, whereas in one case the diagnosis could be narrowed down to a neuroendocrine tumor. No certain immunohistochemical profile with prognostic significance could be identified. It was concluded that immunohistochemistry should be routinely used in cases of undifferentiated carcinoma of unknown primary origin to verify the histological diagnosis and to select the appropriate therapy.
\end{abstract}

A. van der Gaast $\cdot$ J. Verweij $\cdot$ A. S. Th. Planting $\cdot$ G. Stoter Department of Medical Oncology, Rotterdam Cancer Institute, Dr. Daniel den Hoed Cancer Center, Rotterdam, The Netherlands

A. van der Gaast $(\bowtie) \cdot$ J. Verweij · A. S. Th. Planting - G. Stoter Department of Medical Oncology,

University Hospital Rotterdam-Dijkzigt,

Dr. Molewaterplein 40, 3015 GD Rotterdam,

The Netherlands

S. C. Henzen-Logmans

Department of Pathology, Rotterdam Cancer Institute,

Dr. Daniel den Hoed Cancer Center, Rotterdam, The Netherlands
Key words Carcinoma of unknown origin . Immunohistochemistry - Chemotherapy

Abbreviations $\beta H C G \quad \beta$-human chorionic gonadotropin $\cdot A F P \alpha$-fetoprotein $\cdot C E A$ carcinoembryonic antigen $\cdot P S A$ prostate-specific antigen $\cdot P Z F$ prostatic acid phosphatase

\section{Introduction}

Malignant tumors are traditionally classified according to site of origin, tissue type, and histological subtype. Consequently, a variety of tumors can be recognized, each with its specific biological behavior and prognosis. The choice of treatment is mainly related to the site of origin of the tumor and to the tissue type, especially where patients with metastatic malignancies are concerned.

In $0.5 \%-15 \%$ of the cases presenting with metastatic tumors the primary tumor site is not found, despite extensive diagnostic procedures (Holmes and Fouts 1970; Didolkar et al. 1977; Nystrom et al. 1979; Woods et al. 1980; Neumann and Nystrom 1982; Le Chevalier et al. 1988). The prognosis in these cases is generally poor due to poor response to chemotherapy.

Previous studies, both by us and by others, have shown that a subgroup of metastatic carcinomas are chemosensitive, and may therefore have a better prognosis in terms of response rates and survival (Hainsworth et al. 1988; van der Gaast 1990). Some of the characteristics of this subgroup are rapid tumor growth, young age, midline distribution of the tumor and poor histological differentiation. At the Vanderbilt University Medical Center an update on 220 patients with undifferentiated carcinomas or poorly differentiated adenocarcinomas of unknown primary site, treated with cisplatin-containing regimens, showed an objective response in $63 \%$ of the patients, with an actuarial 10 year survival of $16 \%$ (Hainsworth et al. 1992a). 
The clinical utility of immunoperoxidase staining in patients with a light-microscopic diagnosis of undifferentiated carcinomas or poorly differentiated adenocarcinomas of unknown primary site was reported by Hainsworth and co-workers in 1991 (Hainsworth et al. 1991). In 14 patients (16\%) out of a group of 87 , immunohistochemistry led to a different diagnosis, including lymphoma and melanoma. In 24 patients the results were inconclusive, but a more specific diagnosis could be made in 7 out of 18 patients by means of electron microscopy performed on the tissue specimens.

This report describes an investigation of the relevance of immunohistochemical analyses performed on the histological specimens in a similar subgroup of patients. The aims of this study were:

1. To verify the light-microscopic classification of the tissue type of the tumor.

2. To assess whether the application of a panel of antibodies contributes to the identification of a primary organ site.

3. To assess the prognostic significance of various immunohistochemical profiles.

\section{Materials and methods}

Immunohistochemical studies were performed on the tissue specimens of 41 patients with a carcinoma of unknown primary origin treated with chemotherapy.

Tumors were considered to be of unknown primary site if, after a thorough history and physical examination, chest $\mathrm{X}$-ray, routine blood morphology and chemistry tests, mammography in women, palpation and visualization of the female pelvic organs and an assessment of tumor markers such as prostate-specific antigen, $\beta$ human chorionic gonadotropin (HCG) and $\alpha$-fetoprotein (AFP), no primary site could be identified.

The treatment regimen consisted of intravenous administration of $20 \mathrm{mg} / \mathrm{m}^{2}$ cisplatin on days $1-5,120 \mathrm{mg} / \mathrm{m}^{2}$ etoposide on days 1,3 , and 5 , and $30 \mathrm{mg}$ bleomycin on days, 2,9 , and 16 . Four cycles were given at 3 -week intervals.

Only patients with a light-microscopic diagnosis of poorly differentiated adenocarcinoma or undifferentiated carcinoma, meeting at least one of the following criteria, were entered (Greco et al. 1986):

1. Age under 50 years (particularly men)

2. Tumor predominantly located in a midline distribution (mediastinum or retroperitoneum), multiple pulmonary nodules or $1 \mathrm{ym}$ phadenopathy

3. Clinical evidence of rapid tumor growth.

A tumor was classified as a poorly differentiated adenocarcinoma when features like rudimentary gland formation and/or intracellular vacuoles with evidence of mucin production were present. If none of these characteristics was found, the tumor was classified as undifferentiated carcinoma.

Tumor response was evaluated by standard WHO criteria (WHO 1979). Duration of survival was calculated from the starting date of chemotherapy. Patient characteristics are listed in Table 1 .
Table 1 Patient charactristics

\begin{tabular}{ll}
\hline No. patients & 41 \\
Sex & \\
Female & $23(56 \%)$ \\
Male & $18(44 \%)$ \\
Median age (years) & 50 \\
Range & $21-61$ \\
Median performance (WHO) & 1 \\
$\quad$ Range & $0-2$ \\
Histology & \\
Poorly differentiated adenocarcinoma & $23(56 \%)$ \\
$\quad$ Undifferentiated carcinoma & $18(44 \%)$ \\
Response & \\
Complete response & $5(12 \%)$ \\
Partial response & $17(41 \%)$ \\
Stable disease & $12(29 \%)$ \\
Progressive disease & $7(17 \%)$ \\
\hline
\end{tabular}

Immunohistochemistry

Immunohistochemical staining was performed on paraffin sections, using the indirect immunoperoxidase method. Staining results were recorded as either positive or negative for each case. A positive control section was included in each staining series. Phosphatebuffered saline, non-immune ascites fluid or normal rabbit immunoglobulin were used as negative controls (Henzen-Logmans et al. 1987).

In order to verify the tumor tissue type, the following antibodies were selected in appropriate dilutions: monoclonal antibody $(\mathrm{mAb})$ CAM 5.2 (cytokeratin 8,18) (Becton Dickinson, USA); mAB 2662 (broad-spectrum keratin) (DakoPatts, Denmark); polyclonal pVi (vimentin) (Eurodiagnostics Organon Teknika, The Netberlands); $\mathrm{mAb}$ NKI/C3 against melanoma-associated antigen glycoprotein 90-34 (kindly provided by Dr. Claus Vennegoor, NCI, Amsterdam, The Netherlands); DAKO-LCA (CD45) (DakoPatts, Denmark).

In order to identify the primary tumor site, the following tumorselective or tumor-associated antibodies were used: $\mathrm{mAb} C E A$ (carcinoembryonic antigen) (Eurodiagnostics Organon Teknika, The Netherlands); $\mathrm{mAb}$ OV632 against ovarian-associated antigen, (Monosan 9003; Sambio, Uden, The Netherlands); and mAb A577 (calcitonin), A251 (thyroglobulin), A008 (AFP), A231 ( $\beta$ HCG), A627 (PZF), L1838 (PSA) all purchased from DakoPatts, Denmark; and mAb OC125 (HistoCIS, France).

\section{Statistical methods}

The following factors were analyzed for prognostic significance: histology and immunohistochemical staining for cytokeratins, vimentin, NKI/C3, CEA, OV632, calcitonin, thyroglobin, AFP, $\beta \mathrm{HCG}, \mathrm{PZF}$ and PSA. Survival curves were calculated according to the Kaplan-Meier method (Kaplan and Meier 1958) and differences between the survival curves were tested using the log-rank test (Mantel 1966). A $P$-value below 0.05 was considered to be significant.

\section{Results}

Patients with a revised tissue type or definitive diagnosis

Of the 41 patients, 39 had tumors reactive to the anti-cytokeratin antibodies, which suggests epithelial 
differentiation of the tumor. The tumor of 1 of the 2 remaining patients reacted with the anti-leukocyte common antibody (LCA). This patient was referred to our department with a diagnosis of undifferentiated carcinoma but, after additional immunohistochemical studies, proved to have a malignant large-cell nonHodgkin lymphoma. He had a complete response after four cycles of chemotherapy and, more than 5 years after the initial diagnosis, the patient is now diseasefree. The other cytokeratin-negative patient had a tumor that only reacted with anti-vimentin. After additional electron microscopy a tentative diagnosis of neuroblastoma was made. This latter patient achieved a partial response to chemotherapy for 12 months, and survived for 18 months. Apart from the one abovementioned patient no other tumors reacted with anti-LCA.

One tumor (in a 45-year-old patient) reacted with L1838, which is directed against prostate-specific antigen (PSA). This patient presented with a poorly differentiated adenocarcinoma with bone metastases and rapidly growing lymph node metastases in the neck. Rectal examination was initially reported to be negative. On reception of the immunology results a histological biopsy of the prostate was performed, revealing a carcinoma of the prostate, which also turned out to be PSA-positive.

Patients with tumors positive for one or more tumor-associated antigens

In 1 patient the tumor stained with anti-calcitonin. This patient presented with rapidly growing liver metastases of unknown origin. There was no clinical evidence of a (medullary) carcinoma of the thyroid. The initial histological findings were compatible with a poorly differentiated adenocarcinoma. Additional staining revealed that this tumor also expressed neuron-specific enolase and chromogranine $\mathrm{A}$, a reaction pattern characteristic for a neuroendocrine tumor. The patient did not respond to chemotherapy and died 3 months after start of treatment.

In the female patients, immunohistochemical studies with OV-632 and OC-125 monoclonal antibodies, directed against non-mucinous ovarian carcinomas, were performed. None of the tumors expressed OV-632 and only one tumor reacted with OC-125. Post-mortem examination in this patient did not reveal any primary tumor, ovarian or otherwise.

The tumors of 2 patients were $\beta$ HCG-positive. One of these was the previously mentioned patient with prostate cancer. The other patient was a woman who presented with axillary and supraclavicular lymph node metastases of a poorly differentiated adenocarcinoma without any evidence of a germ-cell tumor, apart from the immunology result. This patient partially responded to chemotherapy and was still alive 28 months after start of treatment without evidence of disease progression.

Immunohistochemical patterns requiring further investigations

In 17 patients the tumors stained with NKI/C3. $\mathrm{NKI} / \mathrm{C} 3$ reacts with an antigen, glycoprotein 90-34, expressed in the cytoplasm of melanoma cells. However, like other melanoma-associated antigens, $\mathrm{NKI} / \mathrm{C} 3$ reactivity may also be present in a variety of other tumors (Vennegoor et al. 1985). NKI/C3 often reacts with neuroendocrine tumors and also with mucin-producing tumors of the breast and lung. Especially degenerating cells are often NKI/C3-positive. In 3 patients the tumors stained with cytokeratins as well as with vimentin and $\mathrm{NKI} / \mathrm{C} 3$. As melanomas normally express vimentin but may also express cytokeratin (Zarbo et al. 1990), additional studies were performed in these 3 patients. Electron microscopy performed on the material of 1 patient showed no premelanosomes or melanosomes. Additional staining for S-100 protein and melanin was negative in the 2 other cases. In none of the 17 patients could melanoma be established as the primary diagnosis.

Prognostic relevance of certain immunohistochemical profiles

A total of 16 patients had tumors that stained with anti-CEA. Of these 16 cases, 14 had a histological diagnosis of poorly differentiated adenocarcinoma. The clinical diagnosis could not be narrowed down in any of these cases. No difference in survival $(P=0.64)$ was observed between the 16 patients with CEA-positive tumors and the 25 CEA-negative ones. Likewise, no

Table 2 Prognostic significance of histology and certain immunohistochemical profiles

\begin{tabular}{llll}
\hline Variable & $\begin{array}{l}\text { Response } \\
\text { rate }(\%)\end{array}$ & $\begin{array}{l}\text { Median } \\
\text { survival } \\
\text { (months) }\end{array}$ & $\begin{array}{l}\text { Significance: } \\
P\end{array}$ \\
\hline $\begin{array}{l}\text { Histology } \\
\text { Adenocarcinoma } \\
\quad(n=23)\end{array}$ & 39 & 6 & 0.23 \\
$\quad$ Undifferentiated & 72 & 9 & \\
carcinoma $(n=18)$ & & & \\
Vimentin expression & & & \\
$\quad$ Positive $(n=7)$ & 57 & 6 & 0.49 \\
$\quad$ Negative $(n=34)$ & 51 & 8 & \\
NKI/C3-expression & & & \\
$\quad$ Positive $(n=17)$ & 47 & 5 & 0.08 \\
$\quad$ Negative $(n=24)$ & 58 & 8 & \\
CEA-expression & & & \\
Positive $(n=16)$ & 50 & 7 & \\
$\quad$ Negative $(n=25)$ & 56 & 6 & \\
\hline
\end{tabular}




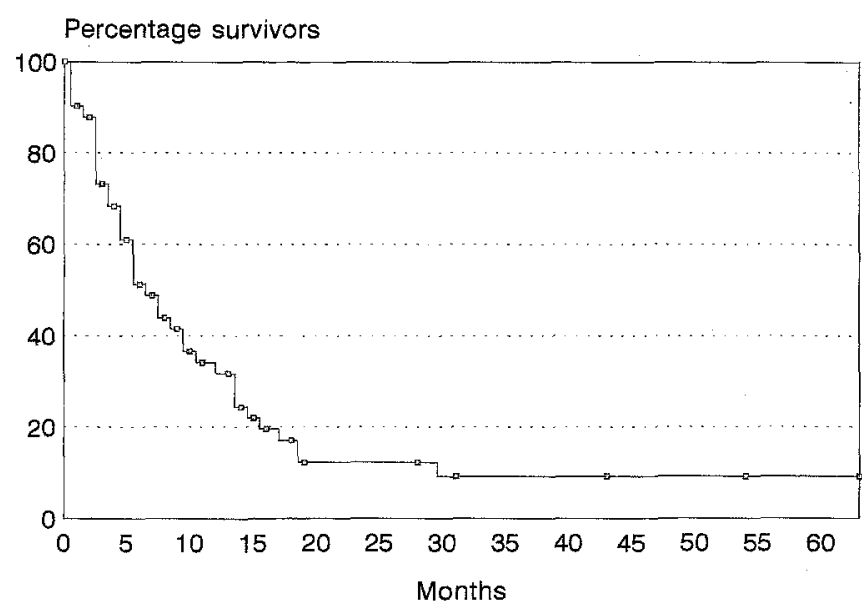

Fig. 1 Survival curve for all patients $(n=41)$

survival difference $(P=0.51)$ was observed between the 7 vimentin-positive cases and the 34 vimentin-negative ones, or between the 17 patients with NKI/C3-positive tumors and the $24 \mathrm{NKI} / \mathrm{C} 3$-negative patients $(P=0.08)$. The influence of the various parameters on response and survival is shown in Table 2 . No discrepancies could be found in the immunoprofiles of the patients with a survival of less than 12 months, as opposed to those with a survival of more than 12 months after the start of treatment. The survival curve for all patients is shown in Fig. 1 .

\section{Discussion}

Despite the development of sophisticated examination methods, such as computed tomography and magnetic resonance imaging, the detection of the primary site of a metastatic solid malignancy remains a problem. It is well known that patients with carcinoma of unknown primary site carry a poor prognosis, not only because the majority of them present with far advanced disease with extensive bone or liver metastases, but also because most tumors are refractory to drug therapy (Ultmann 1991). However, a subset of patients does have a better prognosis after treatment with cisplatincontaining chemotherapy. These patients are characterized by features such as rapid tumor growth, young age, midline distribution of the tumor, and an undifferentiated histology (Hainsworth et al. 1988, 1992b; van der Gaast et al. 1990).

We have investigated the value of immunohistochemical analysis of metastatic tumor tissue in this subgroup of patients. As a result, in 2 patients $(5 \%)$, the tissue diagnosis was changed to a malignant lymphoma and neuroblastoma respectively. In 1 patient with a poorly differentiated calcitonin-positive adenocarcinoma, the diagnosis could be refined to a neuro- endocrine tumor. The primary organ site could be diagnosed in 1 patient with prostate cancer.

In the previously mentioned study of Hainsworth et al. the tissue diagnosis had to be changed in 17 of the 87 patients $(20 \%)$, while in another 4 patients a diagnosis of the primary organ site or a diagnosis refinement could be made (Hainsworth et al. 1991). The low percentage of changed diagnoses in our study may be due to the fact that the majority of our patients were referred from other centers, and that a light-microscopic review of the pathology of the tissue specimens had taken place before the patients entered this study.

In the group of patients we studied, no histological or immunohistochemical characteristics could be identified to discriminate between good or poor prognosis. Although the response rate to chemotherapy was significantly $(P=0.03)$ higher in patients with undifferentiated carcinomas $(72 \%)$ than in patients with poorly differentiated adenocarcinomas $(39 \%)$, the survival curves of these two groups are similar. Greco et al. also found a higher response rate in patients with undifferentiated carcinomas compared to patients with poorly differentiated adenocarcinomas of unknown origin treated with cisplatin-containing chemotherapy. However, in a multivariate analysis, histology was not shown to have an independent predictive value for survival (Greco and Hainsworth 1990; Hainsworth et al. 1992b).

We found more adenocarcinomas than undifferentiated carcinomas to be CEA-positive, as was to be expected (Goslin et al. 1981), but CEA expression had no prognostic significance for either response or survival. Some reports suggest that vimentin expression may be a poor prognostic indicator (Raymond and Leong 1989; Domagala et al. 1990), but the patients' survival pattern in our study showed no correlation with vimentin expression. None of the tested antibodies, or combination of antibodies, appeared to yield a subgroup of patients with prognostic benefit.

The results of this study confirm that a pathology review, including light microscopy and immunohistochemistry, will elucidate the precise tissue and primary organ site diagnosis in about $5 \%$ of cases, and support the contention that immunohistochemistry with a restricted panel of antibodies, such as applied here, should be routinely used in cases of undifferentiated carcinoma of unknown primary origin to verify the histological diagnosis and to select the appropriate therapy.

Acknowledgement Supported by grant CKVO 87-21 of the Netherlands Cancer Foundation.

\section{References}

Didolkar MS, Fanous N, Elias EG, Moore RH (1977) Metastatic carcinomas from occult primary tumor: a study of 254 patients. Ann Surg 186:625-630 
Domagala W, Lasota J, Bartowiak J, Weber K, Osborn M (1990) Vimentin is preferentially expressed in human breast carcinomas with low estrogen receptor and high Ki-67 growth fraction. Am J Pathol 136:219-227

Gaast A van der, Verweij J, Henzen-Logmans SC, Stoter G (1990) Carcinoma of unknown primary: identification of a treatable subset? Ann Oncol 1:119-122

Goslin R, O'Brien MJ, Steele G, Mayer R, Wilson R, Corson JM, Zamcheck N (1981) Correlation of plasma CEA and CEA tissue staining in poorly differentiated colorectal carcinoma. Am J Med $71: 246-253$

Greco FA, Hainsworth JD (1990) Carcinoma of unknown primary site. Ann Oncol 1:98-99

Greco FA, Vaughn WK, Hainsworth JD (1986) Advanced poorly differentiated carcinoma of unknown primary site: recognition of a treatable syndrome. Ann Intern Med 104:547-553

Hainsworth JD, Dial TW, Greco FA (1988) Curative combination chemotherapy for patients with advanced poorly differentiated carcinoma of unknown primary site. Am J Clin Oncol 11: 138-145

Hainsworth JD, Wright EP, Johnson DH, Davis BW, Greco FA (1991) Poorly differentiated carcinoma of unknown primary site: Clinical usefulness of immunoperoxidase staining. J Clin Oncol 9:1931-1938

Hainsworth JD, Johnson DH, Greco FA (1992a) Cisplatin-based combination chemotherapy in the treatment of poorly differentiated carcinoma and poorly differentiated adenocarcinoma of unknown primary site: results of a 12 -year experience. J Clin Oncol 10:912-922

Hainsworth JD, Johnson DH, Greco FA (1992b) The role of cisplatin/bleomycin-based chemotherapy in the treatment of poorly differentiated carcinoma of unknown primary site. Semin Oncol 19 [Suppl 5]:54-58

Henzen-Logmans SC, Mullink H, Vennegoor C, Hilgers J, Oort J, Meijer JLM (1987) Classification of routinely processed anaplastic large cell tumours with a small panel of antibodies. An immunohistochemical study with clinical follow-up. Histol Histopathol 2:107-118
Holmes FF, Fouts TL (1990) Metastatic cancer of unknown primary site. Cancer 26:816-820

Kaplan EL, Meier P (1958) Nonparametric estimation from incomplete observations. J Am Statist Assoc 53:457-481

Le Chevalier T, Cvitkovic E, Caille P, Harvey J, Contesso G, Spielman N, Rouesse J (1988) Early metastatic cancer of unknown primary origin at presentation: a clinical study of 302 consecutive autopsied patients. Arch Intern Med 148: 2035-2039

Mantel N (1966) Evaluation of survival data and two new rank order statistics arising in its consideration. Cancer Chemother Rep 50:163-170

Neumann KH, Nystrom JS (1982) Metastatic cancer of unknown origin; nonsquamous cell type. Semin Oncol 4:427-434

Nystrom JS, Weiner JM, Wolf RM, Bateman JR, Viola MV (1979) Identifying the primary site in metastatic cancer of unknown origin: inadequacy of roentgenographic procedures. JAMA 241:381-383

Raymond WA, Leong A S-Y (1989) Vimentin-a new prognostic parameter in breast carcinoma? J Pathol 158:107-114

Ultmann JE (1991) Cancer of unknown primary site. J Cancer Res Clin Oncol 117:505-509

Vennegoor C, Calafat J, Hageman Ph, van Buitenen F, Janssen $\mathrm{H}$, Kolk A, Rumke Ph (1985) Biochemical characterization and cellular localization of a formalin-resistant melanoma-associated antigen reacting with monoclonal antibody NKI/C-3. Int J Cancer 35:287-295

WHO (1979) Handbook of reporting results of cancer treatment: WHO offset Publ 48

Woods RL, Fox RM, Tattersall MHN, Levi JA, Brodie GN (1980) Metastatic adenocarcinomas of unknown primary: a randomized study of two combination-chemotherapy regimens. N Engl J Med 303:87-89

Zarbo RJ, Gown AM, Nagle RB, Visscher DW, Crissman JD (1990) Anomalous cytokeratin expression in malignant melanoma: one- and two-dimensional Western blot analysis and immunohistochemical survey of 100 melanomas. Mod Pathol $3: 494-501$ 